\title{
L-theanine Prevents Progression of Nonalcoholichepatic Steatosis by Regulating Hepatocyte Lipid Metabolic Pathways via the CaMKKß-AMPK Signaling Pathway
}

Juanjuan Liang

Nanjing Normal University

Lili Gu

Nanjing Normal University

Xianli Liu

Nanjing Normal University

Xintong Yan

Nanjing Normal University

Xiaowen Bi

Nanjing Normal University

Xirui Fan

Nanjing Normal University

Jinyi Zhou

Nanjing Normal University

Shuai Lu

Nanjing Normal University

Lan Luo

Nanjing University

Zhimin Yin ( $\nabla$ yinzhimin@njnu.edu.cn )

Nanjing Normal University

Research

Keywords: L-theanine, hepatic steatosis, lipid accumulation, CaMKK $\beta$, SREBP-1c

Posted Date: September 17th, 2021

DOI: https://doi.org/10.21203/rs.3.rs-820686/v1

License: (c) (1) This work is licensed under a Creative Commons Attribution 4.0 International License.

Read Full License 
Page $2 / 26$ 


\section{Abstract}

Background: L-theanine, a non-protein amino acid found principally in the green tea, has been previously shown to possess potent anti-obesity property and hepatoprotective effect. Herein, we investigated the effects of L-theanine on alleviating nonalcoholic hepatic steatosis in vitro and in vivo, and explored the underlying molecular mechanism.

Methods: In vitro, HepG2 and AML12 cells were treated with $500 \mu \mathrm{M}$ oleic acid (OA) or treated with OA accompanied by L-theanine. In vivo, C57BL/6J mice were fed with normal control diet (NCD), high-fat diet (HFD), or HFD along with L-theanine for 16 weeks. The levels of TG, accumulation of lipid droplets and the expression of genes related to hepatocyte lipid metabolic pathways were detected in vitro and in vivo.

Results: Our data indicated that, in vivo, L-theanine significantly reduced body weight, hepatic steatosis, serum levels of alanine transaminase (ALT), aspartate transaminase (AST), TG and LDL cholesterol (LDLC) in HFD-induced Non-alcoholic fatty liver disease (NAFLD) mice. In vitro, L-theanine also significantly alleviated OA induced hepatocytes steatosis. Mechanic studies showed that L-theanine significantly inhibited the nucleus translocation of sterol regulatory element binding protein 1c (SREBP-1c) through AMPK-mTOR signaling pathway, thereby contributing to the reduction of fatty acid synthesis. We also identified that $L$-theanine enhanced fatty acid $\beta$-oxidation by increasing the expression of peroxisome proliferator-activated receptor a (PPARa) and carnitine palmitoyltransferase-1 A (CPT1A) through AMPactivated protein kinase (AMPK). Furthermore, our study indicated that L-theanine can active AMPK via its upstream kinase Calmodulin-dependent protein kinase kinase- $\beta$ (CaMKK $\beta$ ).

Conclusions: Taken together, our findings suggest that L-theanine alleviates nonalcoholic hepatic steatosis by regulating hepatocyte lipid metabolic pathways via the CaMKKß-AMPK signaling pathway.

\section{Background}

Non-alcoholic fatty liver disease (NAFLD) is the most common chronic liver disease, affecting about a quarter of the global population [1].It characterized by excess triglycerides (TG) accumulation in liver and defined by the presence of steatosis in $>5 \%$ hepatocytes [2]. The pathological spectrum of NAFLD includes non-alcoholic fatty liver (NAFL) and nonalcoholic steatohepatitis (NASH), according to the severity of disease, NASH includes fibrosis, cirrhosis and hepatocellular carcinoma (HCC) [3]. At present, there are no approved therapies for NAFLD, novel therapeutic approach for NAFLD is urgent needed [4].

Recent years, agents derived from natural sources have been widely studied by researchers due to their low toxicity and various biological effects. L-theanine ( $\gamma$-glutamylethylamide) is a non-protein amino acid found principally in the tea plant and mushrooms [5]. It shows beneficial effects on various nutritional and metabolic diseases in human, including antioxidative properties $[6]$. Neuroprotective $[7,8]$ and hepatoprotective [9] effects, anti-tumor effects [10], as well as anti-restenosis effects [11]. L-theanine can reduce the risk of the development of diabetes mellitus type 2 (T2DM) [12]. 
Previous studies have shown that L-theanine can suppress fat accumulation in mice [13], and affect the absorption of lipids by regulating the expression of intestinal fatty acid transporters in rats [14]. Ltheanine also can regulate glucose, lipid, and protein metabolism via insulin and AMP-activated protein kinase (AMPK) signaling pathway in normal rats [15]. Recent studies demonstrate that L-theanine can activate the browning of inguinal white adipose tissue through upregulating the expression of thermogenic genes, thus ameliorates diet-induced obesity in mice [16]. L-theanine also can modulate gut microbiota composition, ameliorated adiposity and hepatic steatosis in obesity mice, they also found that L-theanine can regulate the mRNA expression of genes related to lipid metabolic [17]. however, the above studies did not explore the specific mechanisms underlying the effect of L-theanine on nonalcoholic hepatic steatosis.

Sterol regulatory element-binding protein 1c (SREBP-1c), a key lipogenic transcription factor, is synthesized as an inactive precursor (SREBP-1-P) in endoplasmic reticulum (ER), then it cleaved into nuclear form (SREBP-1-N) in the Golgi apparatus. Ultimately, the SREBP-1-N was translocated to the nucleus [18], and activates transcription of genes related to fatty acid synthesis, include fatty acid synthase (FASN) and acetyl CoA carboxylase 1 (ACC1) [19]. Thus, the trafficking, processing, and transcription of SREBP-1c mediates the pathophysiology of nonalcoholic hepatic steatosis.

In the present study, we demonstrated that L-theanine can decrease fatty acid synthesis through inhibiting the nucleus translocation of SREBP-1c via AMPK-mTOR signaling pathway, L-theanine also can promote the fatty acid the $\beta$-oxidation through AMPK. Moreover, we found that L-theanine can active Calmodulin-dependent protein kinase kinase- $\beta$ (CaMKK $\beta$ ), an upstream kinase of AMPK. Thus, L-theanine ameliorates nonalcoholic hepatic steatosis by regulating hepatocyte lipid metabolic pathways via the CaMKK $\beta$-AMPK signaling pathway, and our study proved that L-theanine may be a novel regent for the treatment of nonalcoholic hepatic steatosis.

\section{Methods}

\section{Materials and reagents}

L-theanine (CAS: 3081-61-6, 98\% purity) were purchased from Sigma-Aldrich (St. Louis, MO, USA). Donkey anti-Rabbit IgG H\&L (Alexa Fluor 488) (\#ab150075; 1:500) was obtained from Abcam (Cambridge, UK). DAPI were purchased from Invitrogen (Carlsbad, CA, USA).

Primary antibodies against ACC1 (\#3676), FASN (\#3180), p-AMPK (\#2535), AMPKa (\#5831) were purchased from Cell Signaling Technology (Beverly, MA, USA). Antibody against p-CaMKK $\beta$ (\#AF4487), CaMKK $\beta$ (\#DF4793) were purchased from Affinity Biosciences (OH, USA). Antibody against SREBP-1c (\#A15586), phospho-mTOR (\#AP0094), mTOR (\#A2445), HRP Goat Anti-Rabbit IgG (\#AS014), and HRP Goat Anti-Mouse IgG (\#AS003) was purchased from ABclonal (Wuhan, Hubei, China). Antibodies against PPARa (\#15540-1-AP), CPT1A (\#15184-1-AP), SREBP-1c (\#14088-1-AP) was purchased from Proteintech (Chicago, IL, USA). Antibodies recognizing GAPDH (\#AP0063), Lamin B (\#AP6001), and B-ACTIN 
(\#AP0060;) were purchased from Bioworld Technology (Minneapolis, MN, USA). Donkey anti-Rabbit IgG H\&L (Alexa Fluor 488) (\#ab150075) was obtained from Abcam (Cambridge, UK). DAPI were purchased from Invitrogen (Carlsbad, CA, USA).

\section{Animals and treatments}

Male C57BL/6J mice weighing 20 to $22 \mathrm{~g}$ ( 6 weeks old) were procured from the Beijing Vital River Laboratory Animal Technology Co. (Beijing, China; SCXK 2019-0001). All animals were kept under standard conditions with a controlled temperature and a 12-h light/dark cycle with water and food ad libitum. After an acclimatization period of 1 week, all mice were randomly divided into 3 groups ( $n=8$ per group). The normal control diet (NCD) group, the high-fat diet (HFD) group (60\% fat, D12492; Research Diets, New Brunswick, NJ, USA), and the L-theanine group (fed with HFD supplement with $300 \mathrm{mg} / \mathrm{kg} \mathrm{L-}$ theanine). The NCD, HFD, and L-theanine group were fed for 16 weeks and in the meanwhile treated with saline or $300 \mathrm{mg} / \mathrm{kg} \mathrm{L}$-theanine by gavage once daily for 16 weeks. Food intake and body weight was recorded weekly. At the end of the experiment, all mice were fasted for $16 \mathrm{~h}$ before sacrifice, then blood samples, liver and visceral adipose tissues were harvested, weighed, and stored at $-80^{\circ} \mathrm{C}$, Additional sections of liver and visceral adipose tissues were prepared for histological analyses.

All treatments of mice in this study were in strict agreement with the "Guide for the Care and Use of Laboratory Animals". All the experimental procedures were approved by the Animal Ethics Committee of Nanjing Normal University.

\section{Biochemical analysis}

The blood samples were collected from orbital vascular plexus and centrifuged at $3000 \mathrm{rpm}$ for $10 \mathrm{~min}$, then serum was collected for biochemical analysis. Serum levels of alanine aminotransferase (ALT), aspartate aminotransferase (AST), HDL cholesterol (HDL-C), LDL cholesterol (LDL-C), total triglycerides (TG) and total cholesterol (TC), were detected using commercial assay kits according to the manufacturer's instructions (Nanjing Jiancheng Bioengineering Institute, Nanjing, China).

TG and TC contents in mouse liver and hepatocytes were also measured using commercial kit (Nanjing Jiancheng Bioengineering Institute, Nanjing, China) according to the manufacturer's protocol.

\section{Oil Red 0 staining}

Liver tissues were fixed with $4 \%$ polyformaldehyde and embedded in tissue freezing medium (Leica). The liver sections $(8 \mu \mathrm{m})$ and treated hepatocytes were immersed in Oil Red O working solution (SigmaAldrich) for 20-30 min at room temperature, then washed with $60 \%$ isopropanol to remove unbound dye and counterstained with hematoxylin. Finally, images were captured by using a light microscope (Nikon, Japan).

\section{Histology and Immunohistochemistry}

Liver and adipose tissues were fixed in $4 \%$ paraformaldehyde and embedded in paraffin. Sections ( $4 \mu \mathrm{m})$ were deparaffinized with xylene and rehydrated in graded ethanol, stained with hematoxylin and eosin 
(H\&E). The images were taken using a microscope (Nikon, Japan), and the NAFLD score was evaluated as previously described [20].

For immunohistochemistry, paraffin sections of liver were deparaffinized and rehydrated, treated for antigen retrieval, and endogenous peroxidase was quenched with $3 \%$ hydrogen peroxide. After blocking nonspecific antigen, the slides were then incubated with primary antibody overnight at $4^{\circ} \mathrm{C}$, followed by biotinylated secondary antibody and third antibody coupled with SABC (BosterTech, China). The DAB kit was used to produce a brown stain, and counterstained with hematoxylin. Images were acquired by using a light microscope (Nikon, Japan).

\section{Cell culture and treatments}

The HepG2 and AML12 cells were purchased from Cell Bank of the Chinese Academic of Sciences (CBCAS), Shanghai, China. HepG2 cells were cultured in EMEM (Wisent, Canada), supplemented with $10 \%$ fetal bovine serum (Wisent, Canada), $100 \mathrm{U} / \mathrm{mL}$ penicillin and $100 \mu \mathrm{g} / \mathrm{mL}$ streptomycin (Wisent). AML12 cells were cultured in DMEM/F12 (Wisent, Canada) supplemented with $10 \%$ fetal bovine serum, 100 $\mathrm{U} / \mathrm{mL}$ penicillin, $100 \mu \mathrm{g} / \mathrm{mL}$ streptomycin, ITS supplement and $40 \mathrm{ng} / \mathrm{mL}$ dexamethasone. All cells were cultured in humidified atmosphere containing $5 \% \mathrm{CO} 2$ at $37^{\circ} \mathrm{C}$ (Thermo Fisher Scientific).

$20 \mathrm{mM} \mathrm{OA}$ solution were obtained by dissolving $\mathrm{OA}$ in $0.1 \mathrm{M} \mathrm{NaOH}$ at $75^{\circ} \mathrm{C}$ in a water bath, then added to $20 \%$ BSA, filtered using $0.22 \mu \mathrm{m}$ filter, a final stock solution of $10 \mathrm{mM} \mathrm{OA}$ were prepared. The $10 \% \mathrm{BSA}$ solution were added as control.

\section{Cell viability assay}

Cell viability was determined using cell counting kit-8 (CCK-8). according to the manufacturer's instructions (Vazyme, Nanjing, China).

\section{Immunofluorescence microscopy}

HepG2 cells grown on coverslips were pretreated with or without L-theanine for 2 hours, after coincubated with $500 \mu \mathrm{M}$ oleic acid (OA) for $24 \mathrm{~h}$, The cells were washed with PBS and fixed with $4 \%$ formaldehyde for $15 \mathrm{~min}$ at room temperature, then cells were permeabilized in $0.2 \%$ TritonX-100 for 20 min followed by blocking with $5 \%$ BSA for $30 \mathrm{~min}$. After that, the cells were incubated anti-SREBP-1c antibody (1:100) overnight at $4^{\circ} \mathrm{C}$ and incubated with Donkey anti-Rabbit IgG H\&L (Alexa Fluor 488) secondary antibody for 1 hour. Cell nuclei were stained with DAPI for $5 \mathrm{~min}$. Images were captured by the Nikon A1 microscope (Tokyo, Japan).

\section{Real-time PCR}

Total RNA from livers was extracted using RNA Isolation Kit (Omega Bio-tek, America) and then was reversely transcribed to cDNA using PrimeScript RT reagent kit (TaKaRa, Shiga, Japan). Quantitative polymerase chain reaction ( $\mathrm{PPCR}$ ) was carried out on ABI StepOnePlus real-time PCR system (Applied Biosystems, USA) using SYBR Premix Ex Taq (TransGen Biotech, China). The results were analysed using 
the $2^{-\Delta \Delta C t}$ method. Values were normalized to $\beta$-actin. The sequences of primers were listed in the Table 1.

Table 1

Primers for Real-Time PCR detection

\begin{tabular}{|c|c|}
\hline Gene & Sequence $5^{\prime} \rightarrow 3^{\prime}$ \\
\hline \multirow[t]{2}{*}{ Mouse Srebf1 } & F: CAAGGCCATCGACTACATCCG \\
\hline & R: CACCACTTCGGGTTTCATGC \\
\hline \multirow[t]{2}{*}{ Mouse Fasn } & F: CCGGAGTCGCTTGAGTATATTG \\
\hline & R: TTGTGGAAGTGCAGGTTAGG \\
\hline \multirow[t]{2}{*}{ Mouse Acc1 } & F: CTTCCTGACAAACGAGTCTGG \\
\hline & R: CTGCCGAAACATCTCTGGGA \\
\hline \multirow[t]{2}{*}{ Mouse Ppary } & F: CTCCAAGAATACCAAAGTGCGA \\
\hline & R: GCCTGATGCTTTATCCCCACA \\
\hline \multirow[t]{2}{*}{ Mouse Ppara } & F: AGAACCTGAGGAAGCCGTTC \\
\hline & R: TTAAGCACGTGCACAATCCC \\
\hline \multirow[t]{2}{*}{ Mouse Cpt1a } & F: TGACGGCTATGGTGTTTCCT \\
\hline & R: TTTTGGAATTGGCGGTGAGG \\
\hline \multirow[t]{2}{*}{ Mouse Actb } & F: GTGACGTTGACATCCGTAAAGA \\
\hline & R: GCCGGACTCATCGTACTCC \\
\hline
\end{tabular}

\section{Preparation of Cytoplasmic and Nuclear Extracts}

Nuclear and cytoplasmic extracts were lysed with NE-PER Nuclear and Cytoplasmic Extraction Reagents (Thermo Fisher Scientific, Rockford, IL, USA) according to the manufacturer's instructions.

\section{Immunoblotting}

Liver samples or cells were lysed in RIPA lysis buffers (Beyotime, China) supplemented with protease inhibitor cocktail and phosphatase inhibitors. A total of 20-50 $\mu \mathrm{g}$ lysates were loaded onto SDS-PAGE gels and transferred to a PVDF membrane (Millipore, China). After blocking with TBS/T (0.1\% Tween-20) containing $5 \%$ nonfat

dry milk for 1 hour at room temperature, the membranes were incubated with primary antibody at $4^{\circ} \mathrm{C}$ overnight followed by HRP-conjugated secondary antibody. Immunoreactive proteins were visualized using the ECL immunoblotting system from Tanon (Shanghai, China).

\section{Statistical analysis}


All data were analyzed using GraphPad Prism 6.0 (GraphPad Software, San Diego, CA). All experimental results are presented as mean \pm SEM. Statistical analysis between two groups were determined using the two-tailed Student's $t$ test. One-way ANOVA followed by the Tukey multiple comparisons test was used for comparison between multiple groups. A $P$ value of less than 0.05 was considered to be statistically significant.

\section{Results}

\section{L-theanine alleviates hepatic steatosis in OA induced HepG2 and AML12 cells}

To elucidate the effects of L-theanine on free fatty acid -induced hepatic steatosis, an in vitro model of was established by stimulating HepG2 and AML12 cells with OA for $24 \mathrm{~h}$. As retains some characteristics of liver cells [21], HepG2 cells are a suitable model to study the lipid metabolism of liver. However, such carcinoma derived cell lines have genetic and characteristic limitations [22]. To overcome its potential shortcomings, we also used mouse hepatocyte cell line AML-12 to rigorously demonstrate the effects of L-theanine on hepatic steatosis.

First, cytotoxicity experiment revealed that L-theanine had no effect on cell viability at the concentration of 1-4 mM (Supplementary Figure. S1A, B). At the concentration of 0-500 $\mu \mathrm{M}, \mathrm{OA}$ also had no effect on cell viability (Supplementary Figure. S1C, D). So, we chose the concentrations of L-theanine (1-4 mM) and $\mathrm{OA}(500 \mu \mathrm{M})$ for subsequent experiments. Next, HepG2 and AML12 cells were treated with $500 \mathrm{uM}$ $\mathrm{OA}$ for $24 \mathrm{~h}$ with or without pretreated L-theanine for $2 \mathrm{~h}$, Oil Red $\mathrm{O}$ staining reveled that lipid droplets were markedly increased in OA group, while L-theanine significantly suppressed intracellular lipid accumulation in a dose-dependent manner (Fig. 1A). Intracellular level of TG in HepG2 (Fig. 1B) and AML12 (Fig. 1C) cells were also remarkably decreased by L-theanine compared with OA group. These results above suggested that L-theanine attenuated steatosis in hepatocyte through decreasing the TG content.

\section{The effects of L-theanine on the metabolism of normal mice}

At first, we explored the effect of L-theanine on mice fed normal control diet (NCD). Mice were randomly divided into four groups $(n=6)$, gavaged with $150,300,600 \mathrm{mg} / \mathrm{kg}$ L-theanine for 16 weeks once daily, saline were gavaged as control. The concentration of L-theanine given to mice was based on previous studies [6, 9]. As result showed, L-theanine had no significant effects on body weight (Supplementary Figure. S2A, B) and the serum levels of ALT, AST, TG, TC, LDL-C (Supplementary Figure. S2C). However, Ltheanine significantly increased the serum level of HDL-C (Supplementary Figure. S2C), and there was a trend that L-theanine decreased the levels of ALT, AST, TG in serum. This indicated the lipid-lowering and hepatoprotective effect of L-theanine on NCD fed mice. Moreover, $300 \mathrm{mg} / \mathrm{kg}$ is a relatively effective dose 
compare to 150 and $600 \mathrm{mg} / \mathrm{kg}$. Hence, subsequent experiments used $300 \mathrm{mg} / \mathrm{kg}$ L-theanine for HFD induced mice.

\section{L-theanine alleviates nonalcoholic nonalcoholic liver steatosis in HFD-induced mice}

Then, an in vivo model of nonalcoholic nonalcoholic liver steatosis was established in HFD induced mice. At the end of experiment, L-theanine significantly decreased body weight which were induced by HFD (Fig. 2A) (Supplementary Figure. S3A, B). Result also suggested that L-theanine significantly reduced the size of inguinal (Supplementary Figure. S3C) and epididymal (Supplementary Figure. S3D) adipose tissue. Furthermore, H\&E-staining performed on adipose tissue sections reveled that L-theanine significantly reduced the average size of inguinal (Supplementary Figure. S3E, G) and epididymal (Supplementary Figure. S3F, H) adipocytes.

The key pathological feature of NAFLD is the accumulation of excess TG in hepatocytes, so we next investigated whether L-theanine can inhibit TG accumulation in the liver. In normal mice, the livers are dark brown-red and have a nearly normal anatomical shape, whereas the livers of HFD mice present light yellow in color and are lackluster due to the accumulation of hepatic lipids. Surprisingly, L-theanine intervention reversed these changes to some extent (Fig. 2B). Histological analysis showed increasing fat vacuoles and lipid droplets in HFD mice, but L-theanine decreased the number and size of lipid droplets (Fig. 2C). The degree of steatosis was graded using activity score of NAFLD, L-theanine treatment group had lower NAFLD activity score compared with HFD group (Fig. 2D). Further, results suggested that Ltheanine decreased hepatic TG content (Fig. 2E), however had no obvious effect on hepatic TC content (Fig. 2F). These above results suggested that L-theanine attenuated nonalcoholic liver steatosis in HFD induced mice. Intervention with L-theanine also decreased ALT, AST, TG, TC, and LDL cholesterol levels in HFD induced mice, However, serum TC and HDL cholesterol levels were not significantly altered by Ltheanine (Fig. 2G).

\section{L-theanine inhibits fatty acid synthesis through down- regulating FASN, ACC1 and PPARY}

To investigate the mechanisms by which L-theanine regulates fatty acid metabolism, we first looked into fatty acid synthesis pathways. Apart from rate-limiting enzymes like AAC and FASN, several fatty acid synthesis/lipolysis genes are regulated by peroxisome proliferator-activated receptor y (PPARY) in liver, and hepatocyte-specific PPARY KO mice are protected from HFD-induced steatosis [23]. In HepG2 and AML12 cells, western blot demonstrated that L-theanine decreased the expressions of FASN, ACC1 and PPARy compared with OA group (Fig. 3A, B). In HFD indued mice, L-theanine significantly decreased the mRNA and protein expressions of FASN, ACC1 and PPARY (Fig. 3C, D), This was further validated by Immunohistochemistry (Fig. 3E). These results demonstrated that L-theanine decreased hepatic TG content through inhibiting the expressions of genes related to fatty acid synthesis. 


\section{L-theanine inhibits fatty acid synthesis through mTOR- SREBP-1C-ACC1/FASN signaling pathway}

As mentioned above, SREBP-1c is a major transcription factor that controls fatty acid synthesis through regulating the transcription of ACC1 and FASN. As the mature form of SREBP-1C is located in the nucleus, we next mainly investigated the effect of L-theanine on the nuclear active form of SREBP-1c (SREBP-1c$\mathrm{N})$. Western blot demonstrated that L-theanine decreased the expression of SREBP-1C-N compared with OA group both in HepG2 and AML12 cells (Fig. 4A, B). In HFD induced mice, L-theanine significantly decreased the mRNA expression of SREBP-1c (Fig. 4C), western blot was consistent with above results (Fig. 4D). the result of Immunohistochemistry also showed that L-theanine decreased the expression of SREBP-1C-N in nucleuses (Fig. 4E).

Immunoblotting of nuclear and cytoplasmic fractions and confocal microscopy imaging were performed to detect the translocation of SREBP-1C. As immunoblotting showed, L-theanine significantly suppressed the expression of SREBP-1 C-N in the nucleus which were increased in OA group (Fig. 5A). Further, Confocal microscopy imaging (Fig. 5B) of SREBP-1c also showed weaker fluorescence of nucleus in Ltheanine group.

As mentioned above, phosphorylation of mTOR promoted the nuclear translocation of SREBP-1c, and our results indicated that L-theanine inhibited the phosphorylation of mTOR in HepG2 and AML12 cells (Fig. $4 A)$.

Taken together, these results confirmed that L-theanine regulated fatty acid synthesis through mTORSREBP-1C-ACC1/FASN pathway.

\section{L-theanine enhances the fatty acid $\beta$-oxidation through promoting the expressions of PPARa and CPT1A}

Apart from the abnormality of fatty acid synthesis, lipid accumulation in liver is also related to $\beta$ oxidation. Next, we investigated the effect of L-theanine on fatty acid $\beta$-oxidation. In HepG2 and AML12 cells, L-theanine significantly promoted the protein expressions of peroxisome proliferator-activated receptor a (PPARa) and carnitine palmitoyltransferase-1 A (CPT1A) (Fig. 6A, B). In mice, L-theanine promoted the mRNA and protein expressions of PPARa and CPT1A compared with HFD group (Fig. 6C, D). As a major transcription factor, PPARa promotes fatty acid $\beta$-oxidation. Immunohistochemistry showed that PPARa had a higher nuclear expression in L-theanine group compared with HFD group (Fig. $6 \mathrm{E})$. These results indicated that L-theanine enhanced fatty acid $\beta$-oxidation through promoting the translocation of PPARa.

\section{L-theanine regulates hepatocyte lipid metabolic pathways via the CaMKK $\beta$-AMPK signaling pathway}


As a cellular energy sensor, AMPK plays an essential role in hepatic lipid metabolism. We next invested the effect of L-theanine on AMPK in OA induced HepG2 cells. Western blot analysis showed that Ltheanine significantly activated AMPK at threonine 172 (Fig. 7A). AMPK is activated by two upstream kinase, LKB1 and CaMKK $\beta$. Previous study demonstrated that L-theanine can activate AMPK through LKB1 in normal rats [15]. Next, we invested whether CaMKK $\beta$ was involved in the activation of AMPK, as results showed, L-theanine activated the phosphorylation of CaMKK $\beta$ in a dose dependent manner (Fig. 7A). cotreatment with a selective CaMKK $\beta$ inhibitor, STO-609, abrogated L-theanine-stimulated AMPK phosphorylation to some extent (Figure. 7B). these data indicated that L-theanine also can activate AMPK through CaMKK $\beta$.

\section{Discussion}

Previous studies have reported that L-theanine can inhibit obesity in mice [13], regulate lipid metabolism in normal rats [15], reduce the risk of T2DM development in human [12]. Recent study indicated that Ltheanine can activate the browning of white adipose tissue [16] and modulation of gut microbiota [17] in obesity mice. In the current study, we found that L-theanine can ameliorates nonalcoholic hepatic steatosis by regulating hepatocyte lipid metabolic pathways via the CaMKKß-AMPK signaling pathway.

Long-term exogenous high-fat diet and overnutrition is the main factor for the development of NAFLD in the clinical condition [24]. Therefore, HFD-induced animal model is used to mimic the pathophysiology of nonalcoholic hepatic steatosis in human. Our results showed that, compared with HFD group, the body weight gain, severity of hepatic steatosis, serum levels of ALT, AST, TG and LDL-C were all decreased in Ltheanine group. Our results were Consistent with previous reports $[16,17]$.

The key pathological feature of hepatic steatosis is the accumulation of excess TG in hepatocytes, and studies had shown that a large part of TG in liver with NAFLD was derived from fatty acid synthesis [25]. Previous studies have shown that L-theanine can decreased the expression of FASN and ACC1 in the liver of normal rats [15], and the mRNA expression in HFD induced liver [17], Our results showed that Ltheanine effectively decreased the mRNA and protein expression of FASN, ACC1 and PPARY in steatosis liver in vitro and in vivo.

FASN and ACC1 are transcriptionally controlled by various transcriptional regulators, especially SREBP-1C [19]. SREBP-1c is synthesized as an inactive precursor in cytoplasm, after the cleavage into the mature form, it shuttles to the nucleus and induces the expression of FASN and ACC1 [18]. In the current study, our data indicated that L-theanine can inhibit the expression of SREBP-1c-N in hepatocytes. furthermore, we found that L-theanine inhibited the nucleus translocation of SREBP-1C.

As a key regulator of cell metabolism and growth in response to nutritional and hormonal stimuli, mTOR deregulation has been implicated in many disease states, including diabetes, obesity and NAFLD. Studies found that mTOR promote the trafficking, processing, and transcription of SREBPs [26]. Previous study showed that mTOR can active SREBP-1c in a S6K1-dependent manner [27], and mTOR also can regulate SREBP-1c through regulating the localization of lipin 1 [28]. Research also showed that mTOR can 
activate SREBP-1c maturation through inhibiting CRTC2 activation, a coactivator that have inhibitory effect on SREBP-1c maturation [29]. In the current study, L-theanine treatment can inhibit the phosphorylation of mTOR which were up-regulated in OA induced hepatocytes. Our data suggested that L-theanine can inhibit the maturation of SREBP-1c through inhibiting phosphorylation of mTOR.

Previous studies showed that the underlying mechanisms of L-theanine action on metabolic disorders appeared to be primarily mediated by the AMPK pathway. Lin et al showed that L-theanine can regulate lipid metabolism through AMPK in the liver of normal rats [15]. Peng et al. showed that L-theanine can regulate a thermogenic programme in white adipose tissue through AMPK [16]. Enhancing the activity of AMPK can prevent and improve nonalcoholic liver steatosis to some extent [30]. As an upstream kinase of mTOR, activation of AMPK can inhibit fatty acid synthesis through down-regulating the activity of mTOR [31]. Our results suggested that L-theanine inhibited fatty acid synthesis through AMPK-mTORSREBP-1C-FASN/ACC1 singling pathway.

Activation of AMPK also can increase fatty acid $\beta$-oxidation [32]. Overexpression of AMPKa1 in the liver increase the expression of CPT1a, which is the rate-limiting enzyme in fatty acid $\beta$-oxidation. Previous studies have shown that L-theanine can increase the mRNA expression of CPT1A in the liver of normal rats [15] or HFD induced mice [17]. Our results indicated that L-theanine can promoted fatty acid $\beta$ oxidation through up-regulating the mRNA and protein expression of PPARa and CPT1A in vivo and in vitro [33]. Our results suggested that L-theanine can activate the AMPK at threonine 172 on the AMPKa subunit.

To date, two AMPK upstream kinases have been characterized, the first is the tumor suppressor liver kinase B1 (LKB1) [34] and the second is Ca2+/ Calmodulin-dependent protein kinase kinase $\beta$ (CaMKK $\beta$ ) [35]. Previous study demonstrated that L-theanine can activate AMPK via its upstream kinases LKB1 in normal rat liver [15]. In the current study we invested another AMPK upstream kinase CaMKKB. Our results demonstrated $\mathrm{L}$-theanine also can activate AMPK through $\mathrm{Ca}^{2+}{ }^{2}-\mathrm{CaMKK} \beta$-mediated pathway. Studies showed that LKB1 can response to a change in the AMP:ATP ratio, thus actives AMPK via AMP, and CaMKK $\beta$ activate AMPK mainly through $\mathrm{Ca}^{2+}$, however, the increase of $\mathrm{Ca}^{2+}$ would also change the AMP:ATP ratio, and vice versa [36]. So, in many cases, the activation of AMPK is the consequence of both LKB1 and CaMKK 3 [37]. Thus, the activation of AMPK mediated by L-theanine may via both LKB1 and CaMKK $\beta$. Our data suggest that the effects of L-theanine may be used to develop a novel regent for the management of nonalcoholic liver steatosis.

\section{Conclusions}

In summary, our experimental data indicated that L-theanine can protect against nonalcoholic hepatic steatosis in vitro and in vivo. Our mechanic studies indicated that L-theanine can decrease fatty acid synthesis through CaMKK $\beta$-AMPK-mTOR-SRBEBP-1c-FASN/ACC1 signaling pathway, at the same time promote fatty acid $\beta$-oxidation through AMPK-PPARa-CPT1A signaling pathway (Fig. 8). We explained the 
specific mechanism by which theanine alleviates nonalcoholic hepatic steatosis, and proved that Ltheanine may be a novel regent for the treatment of nonalcoholic hepatic steatosis.

\section{Abbreviations}

NAFLD: Non-alcoholic fatty liver disease; TG: triglycerides; TC: total cholesterol; ALT: alanine aminotransferase; AST: aspartate aminotransferase; HDL-C: HDL cholesterol; LDL-C: LDL cholesterol; HFD: high-fat-diet; NCD: normal control diet; OA: oleic acid; SREBP-1c: Sterol regulatory element-binding protein-1c; SREBP-1c-P: precursor form of SREBP-1 c; SREBP-1c-N: nuclear active form of SREBP-1c. FASN: fatty acid synthase; ACC1: acetyl CoA carboxylase; PPARY: peroxisome proliferator-activated receptor $\mathrm{Y}$; PPARa: peroxisome proliferator-activated receptor $\alpha$; CPT1A: carnitine palmitoyltransferase-1 A. AMPK: AMP-activated protein kinase; CaMKK $\beta$ : Calmodulin-dependent protein kinase kinase- $\beta$.

\section{Declarations}

\section{Ethics approval and consent to participate}

All treatments of mice in this study were in strict agreement with the "Guide for the Care and Use of Laboratory Animals". All the experimental procedures were approved by the Animal Ethics Committee of Nanjing Normal University.

\section{Consent for publication}

Not applicable.

\section{Availability of data and materials}

The datasets used and/or analysed during the current study are available from the corresponding author on reasonable request.

\section{Competing interests}

The authors declare that they have no competing interests.

\section{Funding}

This work was financially supported by grants from the Natural Science Foundation of China (Nos. 81771703, 81671565 and 31901012), The China Postdoctoral Science Foundation 
(Nos.2020T130058ZX), The Priority Academic Program Development of Jiangsu Higher Education Institution (PADD).

\section{Authors' contributions}

Conception and design: Zhimin Yin, Juanjuan Liang, Lili Gu. Development of methodology: Zhimin Yin, Juanjuan Liang, Lan Luo, Da Zhou. Acquisition of data: Juanjuan Liang, Lili Gu, Xianli Liu. Analysis and interpretation of data: Xintong Yan, Xiaowen Bi, Xirui Fan, Jinyi Zhou, Shuai Lu,. Writing, review and revision of the manuscript: Juanjuan Liang, Zhimin Yin, Jinyi Zhou, Shuai Lu. Study supervision: Zhimin Yin, Lan Luo.

\section{Acknowledgments}

We thank members of the Luo laboratory for their technical support and discussions throughout the study.

\section{References}

1. Younossi Z, Anstee QM, Marietti M, Hardy T, Henry L, Eslam M, et al. Global burden of NAFLD and NASH: trends, predictions, risk factors and prevention. Nature reviews Gastroenterology hepatology. 2018;15:11-20.

2. Chalasani N, Younossi Z, Lavine JE, Charlton M, Cusi K, Rinella M, et al. The diagnosis and management of nonalcoholic fatty liver disease: Practice guidance from the American Association for the Study of Liver Diseases. Hepatology. 2018;67:328-57.

3. EASL-EASD-EASO. Clinical Practice Guidelines for the management of non-alcoholic fatty liver disease. Journal of hepatology. 2016;64:1388-402.

4. Sheka AC, Adeyi O, Thompson J, Hameed B, Crawford PA, Ikramuddin S. Nonalcoholic Steatohepatitis: A Review. Jama. 2020;323:1175-83.

5. Türközü D, Şanlier N. L-theanine, unique amino acid of tea, and its metabolism, health effects, and safety. Critical reviews in food science nutrition. 2017;57:1681-7.

6. Li G, Ye Y, Kang J, Yao X, Zhang Y, Jiang W, et al. I-Theanine prevents alcoholic liver injury through enhancing the antioxidant capability of hepatocytes. Food chemical toxicology: an international journal published for the British Industrial Biological Research Association. 2012;50:363-72.

7. Ben P, Zhang Z, Zhu Y, Xiong A, Gao Y, Mu J, et al. I-Theanine attenuates cadmium-induced neurotoxicity through the inhibition of oxidative damage and tau hyperphosphorylation. Neurotoxicology. 2016;57:95-103.

8. Kim TI, Lee YK, Park SG, Choi IS, Ban JO, Park HK, et al. I-Theanine, an amino acid in green tea, attenuates beta-amyloid-induced cognitive dysfunction and neurotoxicity: reduction in oxidative 
damage and inactivation of ERK/p38 kinase and NF-kappaB pathways. Free Radic Biol Med. 2009;47:1601-10.

9. Gong Z, Liu Q, Lin L, Deng Y, Cai S, Liu Z, et al. I-Theanine prevents ETEC-induced liver damage by reducing intrinsic apoptotic response and inhibiting ERK1/2 and JNK1/2 signaling pathways. Eur $\mathrm{J}$ Pharmacol. 2018;818:184-90.

10. Fan X, Zhou J, Bi X, Liang J, Lu S, Yan X, et al. L-theanine suppresses the metastasis of prostate cancer by downregulating MMP9 and Snail. J Nutr Biochem. 2021;89:108556.

11. Bi A, Hang Q, Huang Y, Zheng S, Bi X, Zhang Z, et al. I-Theanine attenuates neointimal hyperplasia via suppression of vascular smooth muscle cell phenotypic modulation. J Nutr Biochem. 2020;82:108398.

12. Ninomiya T, Kanzaki N, Hirakawa Y, Yoshinari M, Higashioka M, Honda T, et al. Serum Ethylamine Levels as an Indicator of I-Theanine Consumption and the Risk of Type 2 Diabetes in a General Japanese Population: The Hisayama Study. Diabetes Care. 2019;42:1234-40.

13. Zheng G, Sayama K, Okubo T, Juneja LR, Oguni I. Anti-obesity effects of three major components of green tea, catechins, caffeine and theanine, in mice. In vivo (Athens, Greece). 2004;18:55-62.

14. Yan Q, Tong H, Tang S, Tan Z, Han X, Zhou C. L-Theanine Administration Modulates the Absorption of Dietary Nutrients and Expression of Transporters and Receptors in the Intestinal Mucosa of Rats. BioMed research international. 2017;2017:9747256.

15. Lin L, Zeng L, Liu A, Peng Y, Yuan D, Zhang S, et al. I-Theanine regulates glucose, lipid, and protein metabolism via insulin and AMP-activated protein kinase signaling pathways. Food function. 2020;11:1798-809.

16. Peng WQ, Xiao G, Li BY, Guo YY, Guo L, Tang QQ. L-Theanine Activates the Browning of White Adipose Tissue through the AMPK/a-Ketoglutarate/Prdm16 Axis and Ameliorates Diet-induced Obesity in Mice. Diabetes. 2021.

17. He J, Chen J, He Q, Li S, Jian L, Xie F, et al. Oral L-theanine administration promotes fat browning and prevents obesity in mice fed high-fat diet associated with the modulation of gut microbiota. J Funct Food. 2021;81:12.

18. Horton JD, Goldstein JL, Brown MS. SREBPs: activators of the complete program of cholesterol and fatty acid synthesis in the liver. J Clin Investig. 2002;109:1125-31.

19. Wang Y, Viscarra J, Kim SJ, Sul HS. Transcriptional regulation of hepatic lipogenesis. Nature reviews Molecular cell biology. 2015;16:678-89.

20. Kleiner DE, Brunt EM, Van Natta M, Behling C, Contos MJ, Cummings OW, et al. Design and validation of a histological scoring system for nonalcoholic fatty liver disease. Hepatology. 2005;41:1313-21.

21. Mersch-Sundermann V, Knasmüller S, Wu XJ, Darroudi F, Kassie F. Use of a human-derived liver cell line for the detection of cytoprotective, antigenotoxic and cogenotoxic agents. Toxicology. 2004;198:329-40.

22. Kongmanas K, Punyadee N, Wasuworawong K, Songjaeng A, Prommool T, Pewkliang Y, et al. Immortalized stem cell-derived hepatocyte-like cells: An alternative model for studying dengue 
pathogenesis and therapy. PLoS Negl Trop Dis. 2020;14:e0008835.

23. Morán-Salvador E, López-Parra M, García-Alonso V, Titos E, Martínez-Clemente M, González-Périz A, et al. Role for PPARY in obesity-induced hepatic steatosis as determined by hepatocyte- and macrophage-specific conditional knockouts. FASEB journal: official publication of the Federation of American Societies for Experimental Biology. 2011;25:2538-50.

24. Ma Z, Chu L, Liu H, Wang W, Li J, Yao W, et al. Beneficial effects of paeoniflorin on non-alcoholic fatty liver disease induced by high-fat diet in rats. Scientific reports. 2017;7:44819.

25. Friedman SL, Neuschwander-Tetri BA, Rinella M, Sanyal AJ. Mechanisms of NAFLD development and therapeutic strategies. Nat Med. 2018;24:908-22.

26. Han J, Wang Y. mTORC1 signaling in hepatic lipid metabolism. Protein cell. 2018;9:145-51.

27. Owen JL, Zhang Y, Bae SH, Farooqi MS, Liang G, Hammer RE, et al. Insulin stimulation of SREBP-1C processing in transgenic rat hepatocytes requires p70 S6-kinase. Proc Natl Acad Sci U S A. 2012;109:16184-9.

28. Peterson TR, Sengupta SS, Harris TE, Carmack AE, Kang SA, Balderas E, et al. mTOR complex 1 regulates lipin 1 localization to control the SREBP pathway. Cell. 2011;146:408-20.

29. Han J, Li E, Chen L, Zhang Y, Wei F, Liu J, et al. The CREB coactivator CRTC2 controls hepatic lipid metabolism by regulating SREBP1. Nature. 2015;524:243-6.

30. Smith BK, Marcinko K, Desjardins EM, Lally JS, Ford RJ, Steinberg GR. Treatment of nonalcoholic fatty liver disease: role of AMPK. Am J Physiol Endocrinol Metab. 2016;311:E730-e40.

31. Xu J, Ji J, Yan XH. Cross-talk between AMPK and mTOR in regulating energy balance. Critical reviews in food science nutrition. 2012;52:373-81.

32. Day EA, Ford RJ, Steinberg GR. AMPK as a Therapeutic Target for Treating Metabolic Diseases. Trends Endocrinol Metab. 2017;28:545-60.

33. Zhang HA, Yang XY, Xiao YF. AMPKa1 overexpression alleviates the hepatocyte model of nonalcoholic fatty liver disease via inactivating p38MAPK pathway. Biochem Biophys Res Commun. 2016;474:364-70.

34. Shaw RJ, Kosmatka M, Bardeesy N, Hurley RL, Witters LA, DePinho RA, et al. The tumor suppressor LKB1 kinase directly activates AMP-activated kinase and regulates apoptosis in response to energy stress. Proc Natl Acad Sci U S A. 2004;101:3329-35.

35. Hawley SA, Pan DA, Mustard KJ, Ross L, Bain J, Edelman AM, et al. Calmodulin-dependent protein kinase kinase-beta is an alternative upstream kinase for AMP-activated protein kinase. Cell Metabol. 2005;2:9-19.

36. Carafoli E. Calcium-mediated cellular signals: a story of failures. Trends Biochem Sci. 2004;29:3719.

37. Woods A, Dickerson K, Heath R, Hong SP, Momcilovic M, Johnstone SR, et al. Ca2+/calmodulindependent protein kinase kinase-beta acts upstream of AMP-activated protein kinase in mammalian cells. Cell Metabol. 2005;2:21-33. 


\section{Figures}

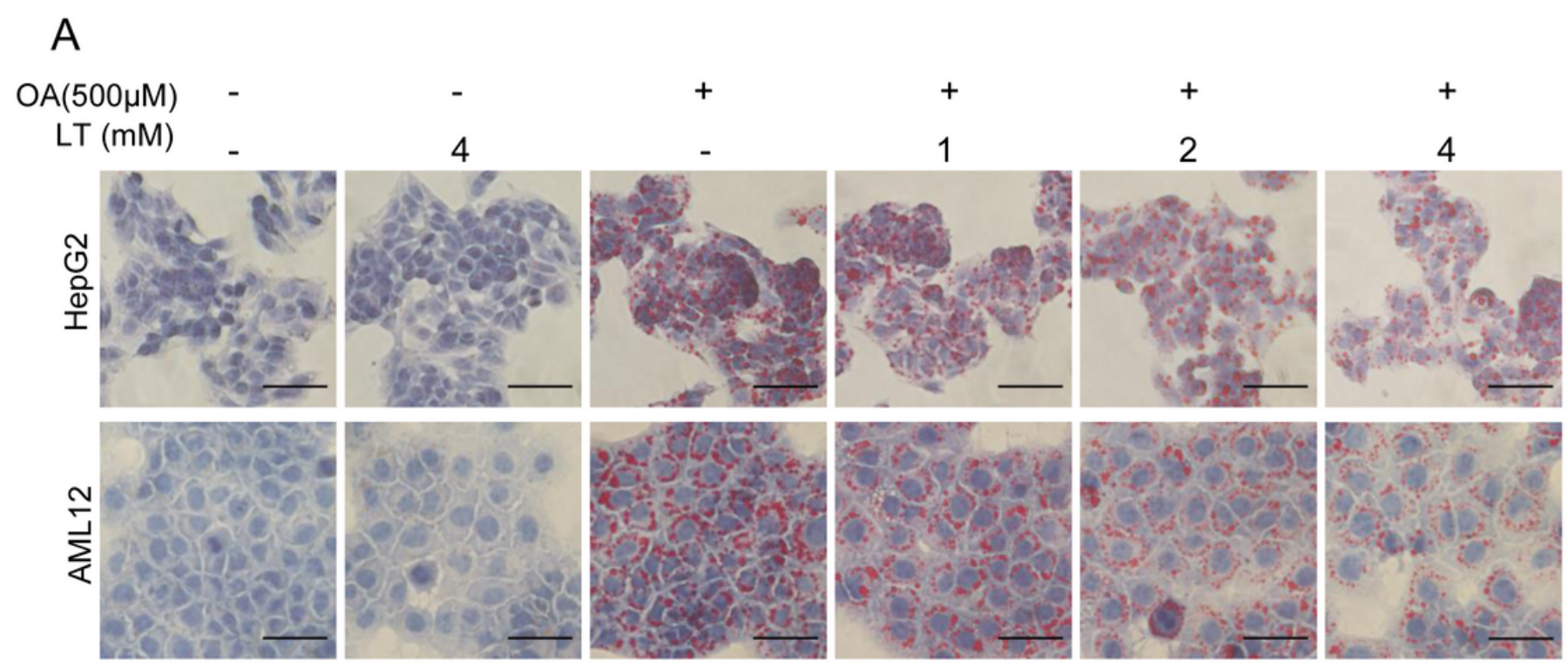

B

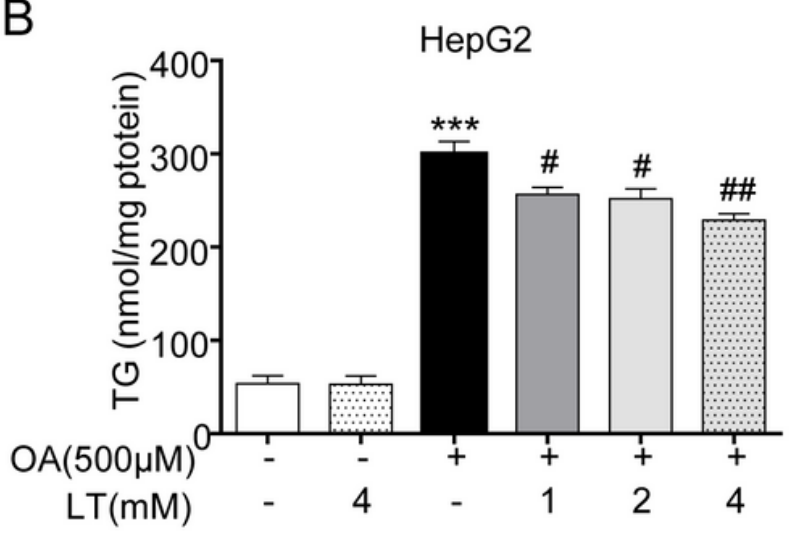

C

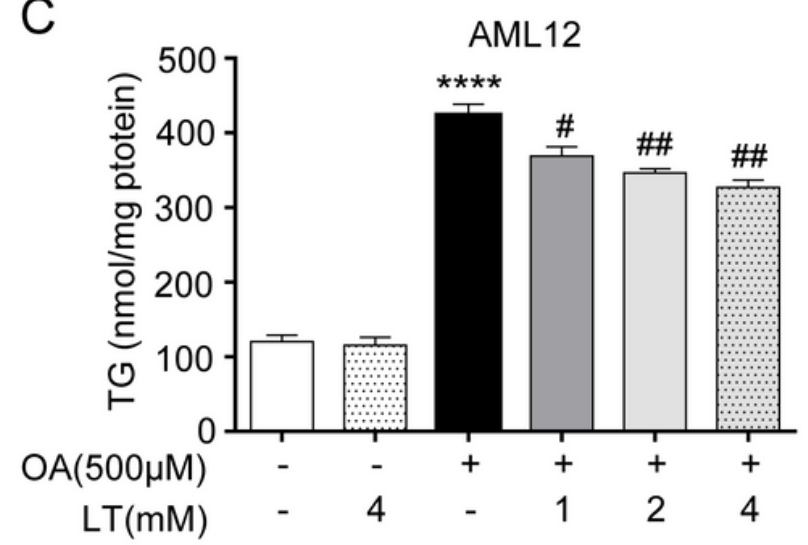

\section{Figure 1}

L-theanine alleviates hepatic steatosis in OA induced HepG2 and AML12 cells. HepG2 and AML12 cells were treated with $500 \mathrm{uM} \mathrm{OA}$ for $24 \mathrm{~h}$ with or without pretreated L-theanine for $2 \mathrm{~h}$. (A) Representative Oil Red $O$ staining of HepG2 and AML12 cells. Intracellular TG levels in HepG2 (B) and AML12 (C) cells. Data shown were representative of three independent experiments. Values are expressed as mean $\pm S E M$. * $p<$ $0.05,{ }^{* \star} p<0.01, * \star \star p<0.001, * \star \star \star p<<0.0001$ VS Control group; $\# p<0.05, \# \# p<0.01$ VS OA group. Scale bar $=50 \mu \mathrm{m}$. Abbreviation: LT: L-theanine; OA: oleic acid. 
A

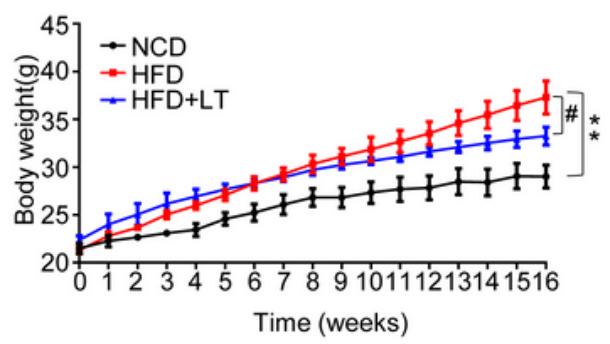

B

C

NCD

HFD
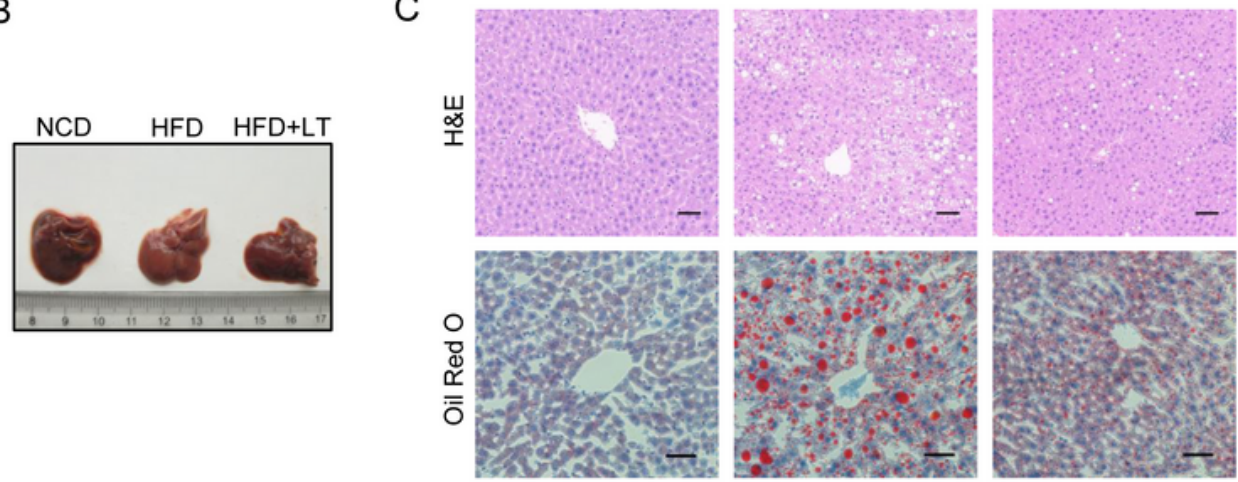

D

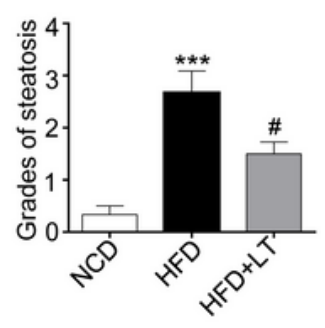

$\mathrm{E}$
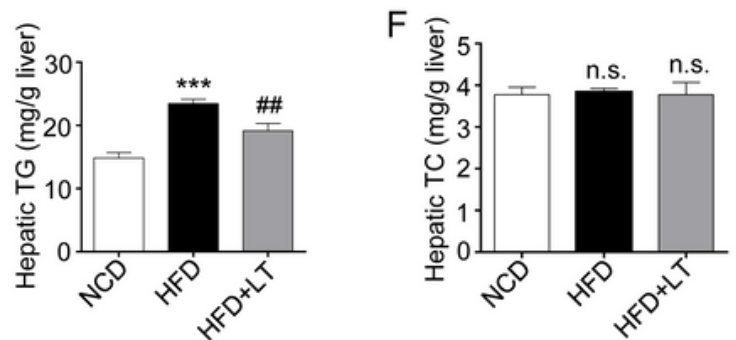

G
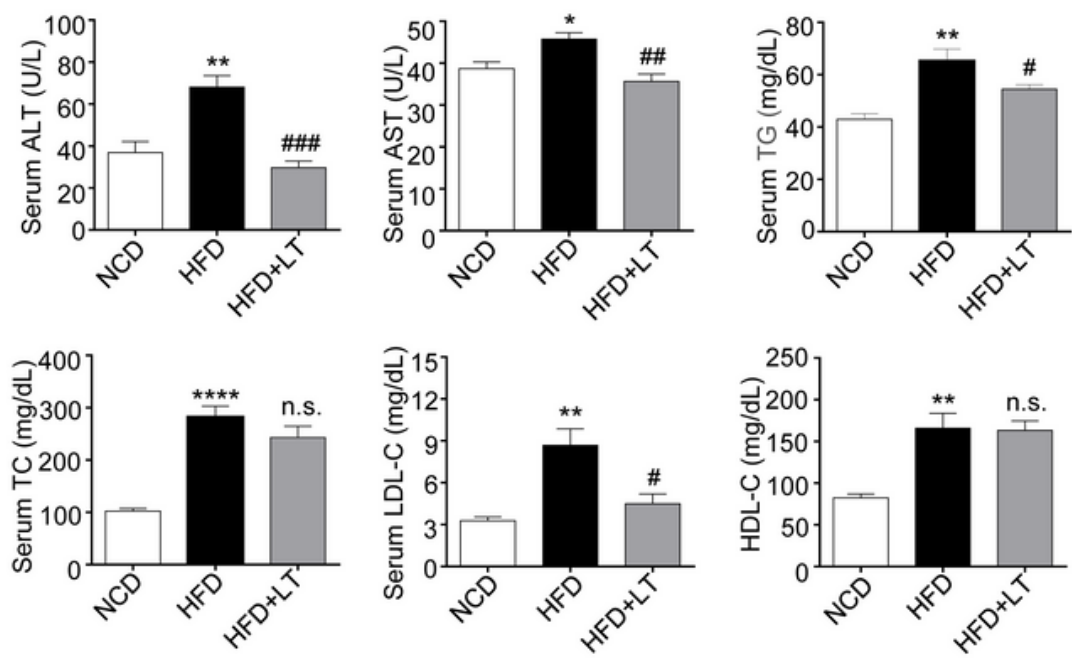

\section{Figure 2}

L-theanine alleviates nonalcoholic liver steatosis in HFD-induced mice. C57BL/6J mice were fed normal control diet (NCD), HFD or HFD supplemented with L-Theanine for 16 weeks, mice were gavaged with $300 \mathrm{mg} / \mathrm{kg}$ L-theanine once a day for 16 weeks, saline were gavaged as control $(n=8)$. (A) Body weight gain curves of mice in indicated groups. (B) Macroscopic images of liver in mice. (C) Representative images of H\&E and Oil Red $O$ staining of liver tissue sections are shown. (D) Activity score of NAFLD. 
Grades of steatosis are as follows: 0 , no steatosis; 1 , mild; 2 , moderate; 3 , severe; 4 , very severe. Hepatic TG (E) and TC (F) contents of liver were measured by commercial kits and expressed as milligrams per gram of tissue $(n=8)$. ( $(G)$ Serum concentrations of ALT, AST, TG, TC, LDL-C and HDL-C in indicated groups. Values are expressed as mean \pm SEM . ${ }^{*} p<0.05$, ${ }^{\star *} p<0.01,{ }^{* \star *} p<0.001,{ }^{* \star * *} p<0.0001$ VS NCD group; \#p < 0.05, \#\#p < 0.01, \#\#\#p < 0.001 VS HFD group. Scale bar $=50 \mu \mathrm{m}$. Abbreviation: LT, Ltheanine. n.s.: not significant.
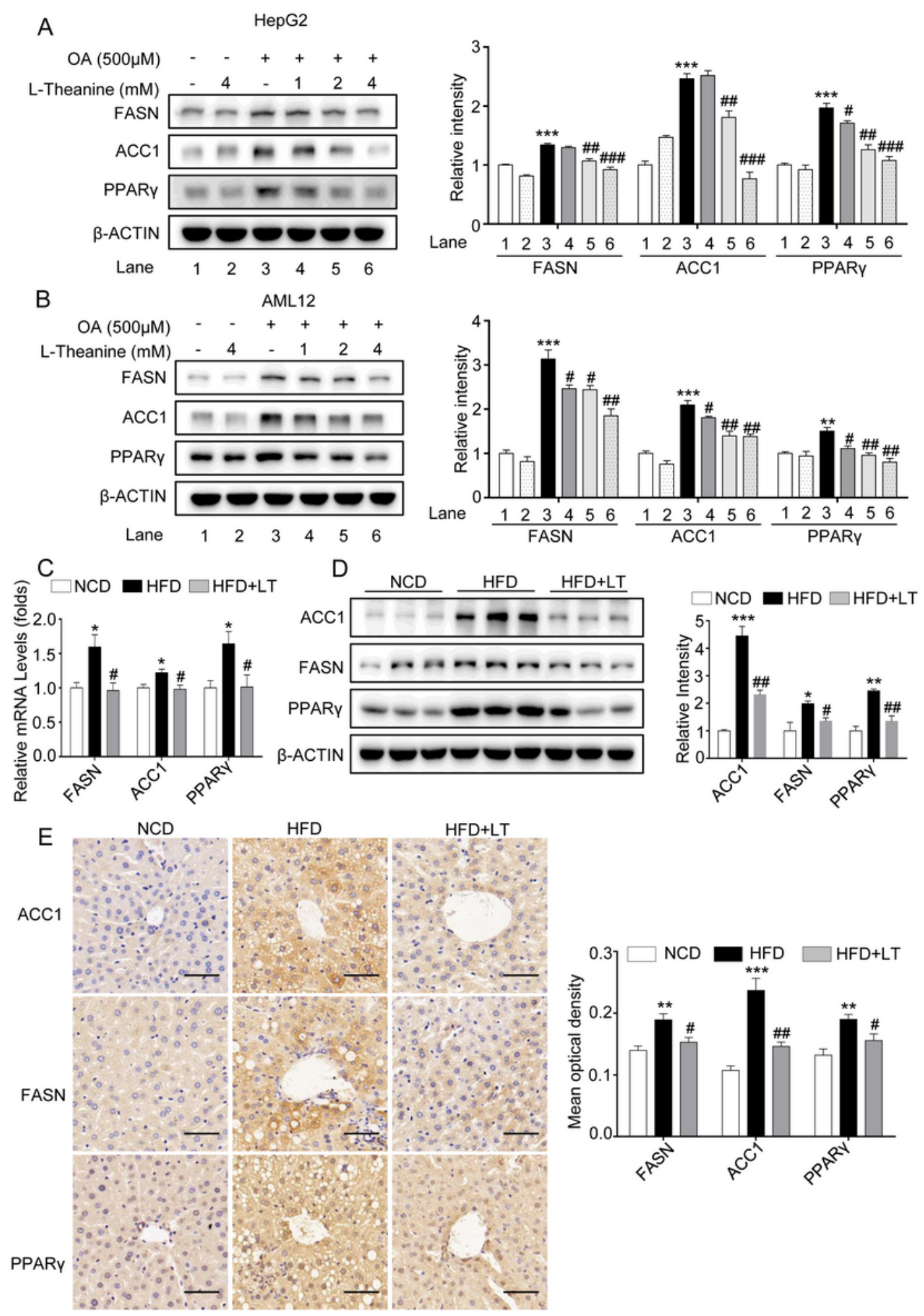

Figure 3 
L-theanine inhibits fatty acid synthesis through down-regulating FASN, ACC1 and PPARY. HepG2 and AML 12 cells were treated with $500 \mathrm{uM} \mathrm{OA}$ for $24 \mathrm{~h}$ with or without pretreated L-theanine for $2 \mathrm{~h}$. Western blot analysis showing protein expression of FASN, ACC1 and PPARy in HepG2 (A) and AML12 (B) cells. C57BL/6J mice were fed normal control diet (NCD), HFD or HFD supplemented with L-Theanine for 16 weeks $(n=8)$. (C) Realtime quantitative PCR analyses the levels of FASN, ACC1 and PPARY mRNA expression in the mice liver. (D) Western blot analysis showing protein expression of FASN, ACC1 and PPARY in mice liver. (E) Representative images of immunohistochemical staining of FASN, ACC1 and PPARy in liver sections. Band intensity was quantified by densitometry analysis. Values are expressed as mean \pm SEM deviation of three independent experiments. ${ }^{*} p<0.05$, ${ }^{* \star} p<0.01,{ }^{* \star *} p<0.001$ VS control or NCD group; \#p < 0.05, \#\#p < 0.01, \#\#\#p < 0.001 VS OA or HFD group. Scale bar $=50 \mu \mathrm{m}$. Abbreviation: LT, L-Theanine. 
A

HepG2
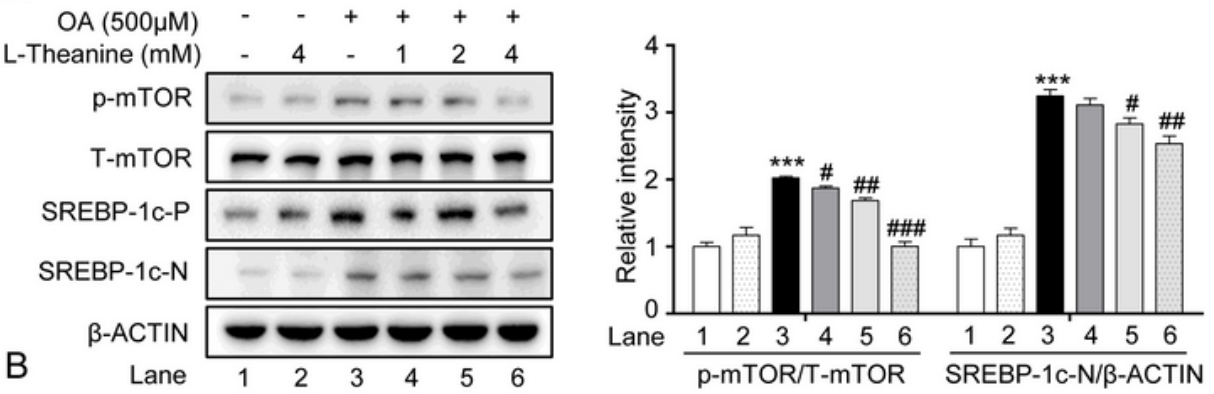

AML12
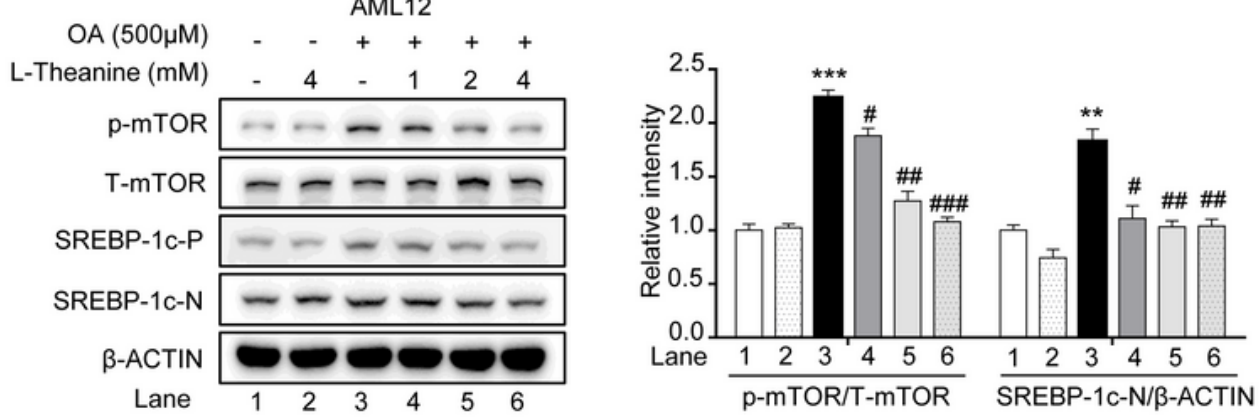

C

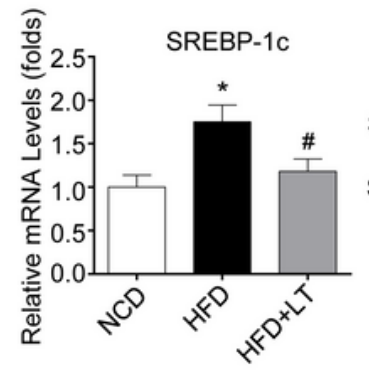

$\mathrm{E}$

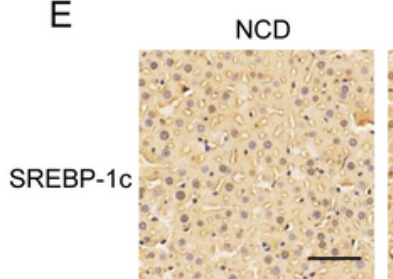

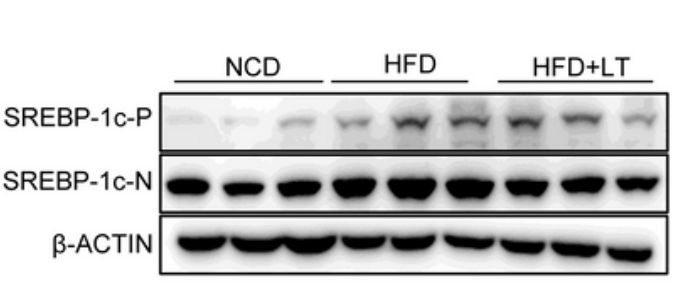

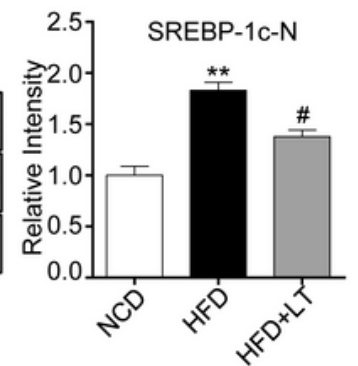

HFD

HFD+LT

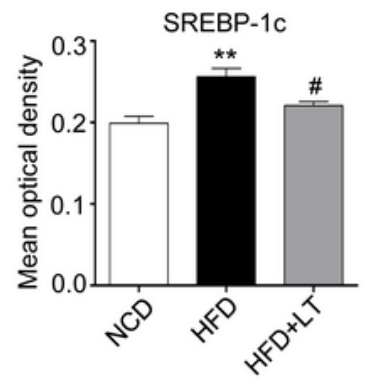

\section{Figure 4}

L-theanine inhibits fatty acid synthesis through mTOR-SREBP1c-ACC1/FASN signaling pathway. HepG2 and AML12 cells were treated with $500 \mathrm{uM} \mathrm{OA}$ for $24 \mathrm{~h}$ with or without pretreated L-theanine for $2 \mathrm{~h}$. Western blot analysis showing protein expression of SREBP -1c, p-mTOR, T-mTOR in HepG2 (A) and AML12 cells (B). C57BL/6J mice were fed normal control diet (NCD), HFD or HFD supplemented with LTheanine for 16 weeks $(n=8)$. (C) mRNA expression of SREBP -1c in mice liver. (D) Protein levels of 
SREBP-1c in mice liver. (E) Representative images of immunohistochemical staining of SREBP -1c in liver sections., Scale bars $=10 \mu \mathrm{m}$. Band intensity was quantified by densitometry analysis. Values are expressed as mean \pm SEM deviation of three independent experiments. ${ }^{*} p<0.05,{ }^{*} p<0.01$, ${ }^{* \star *} p<0.001$ VS control or NCD group; \#p $<0.05$, \#\#p $<0.01$, \#\#\#p $<0.001$ VS OA or HFD group. Scale bar $=50 \mu \mathrm{m}$. Abbreviation: LT: L-Theanine; SREBP-1c-P: precursor form of SREBP-1c; SREBP-1c-N: nuclear active form of SREBP-1c. p-mTOR: phosphorylated levels of mTOR; T-mTOR: tolal levels of mTOR.

\title{
A
}
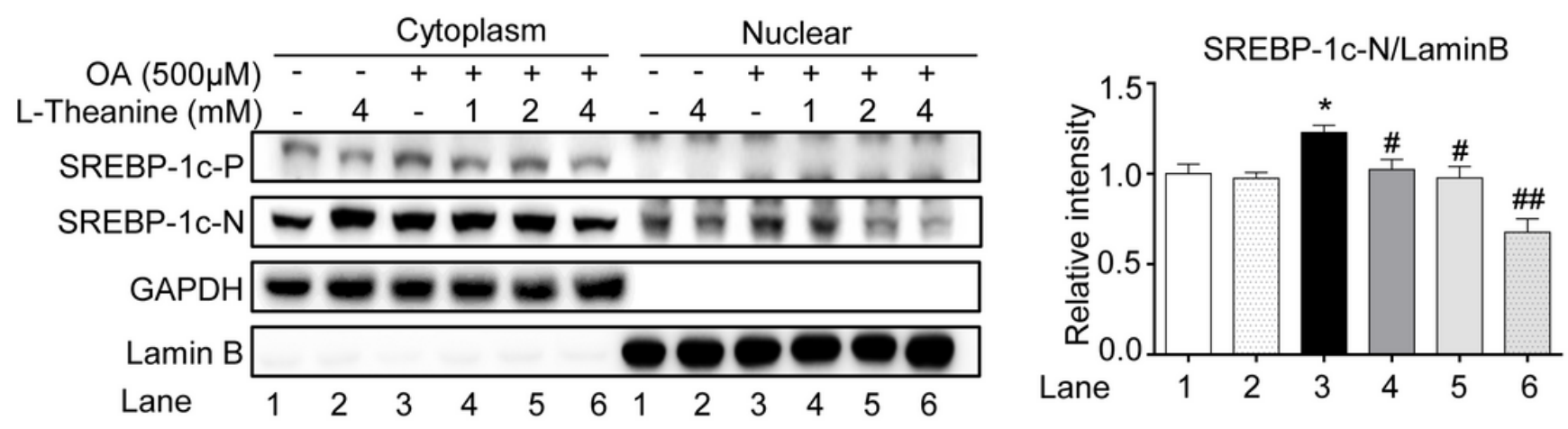

B

\author{
$\mathrm{OA}(500 \mu \mathrm{M})$ \\ L-theanine $(\mathrm{mM})$ \\ SREBP-1C
}
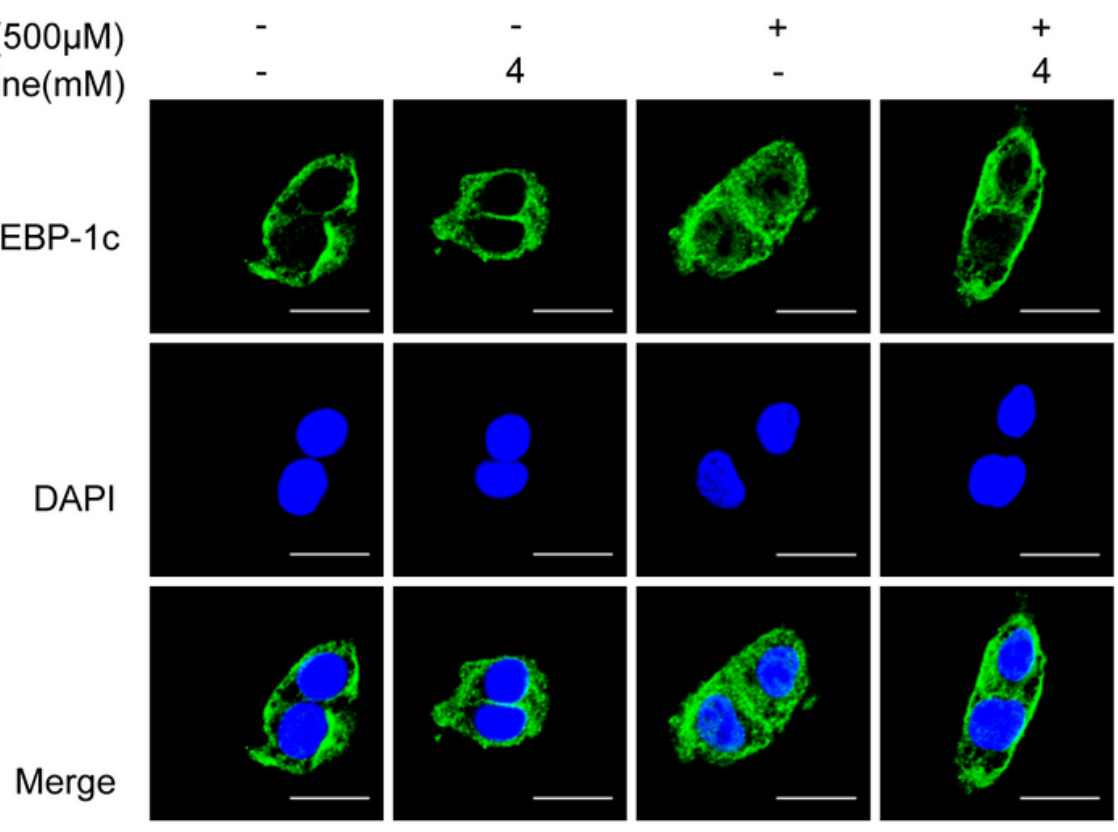

\section{Figure 5}

L-theanine inhibits the nucleus translocation of SREBP-1c. (A) Immunoblotting was performed to detect the level of SREBP-1C in the nucleus and cytoplasm. (B) Immunofluorescence analysis was performed to explore the effect of L-theanine (4 mM) on the nuclear translocation of SREBP-1c in HepG2 cells, original magnification, $60 \times$. Band intensity was quantified by densitometry analysis. Values are expressed as 
mean \pm SEM deviation of three independent experiments. ${ }^{\star} p<0.05$ VS control group; $\# p<0.05$, \#\#p $<$ 0.01 VS OA group. Scale bar $=10 \mu \mathrm{m}$. Abbreviation: LT: L-Theanine; SREBP-1c-P: precursor form of SREBP-1c; SREBP-1c-N: nuclear active form of SREBP-1c.

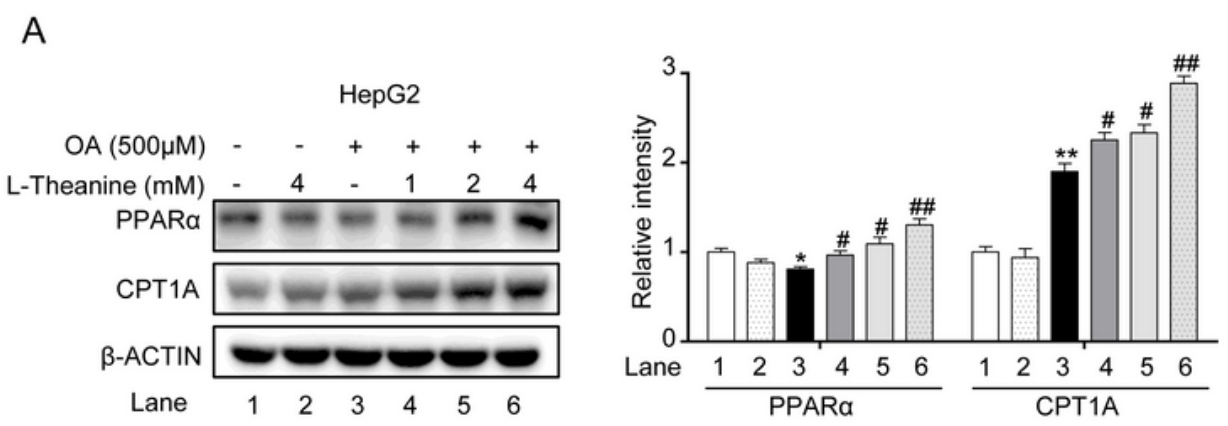

B

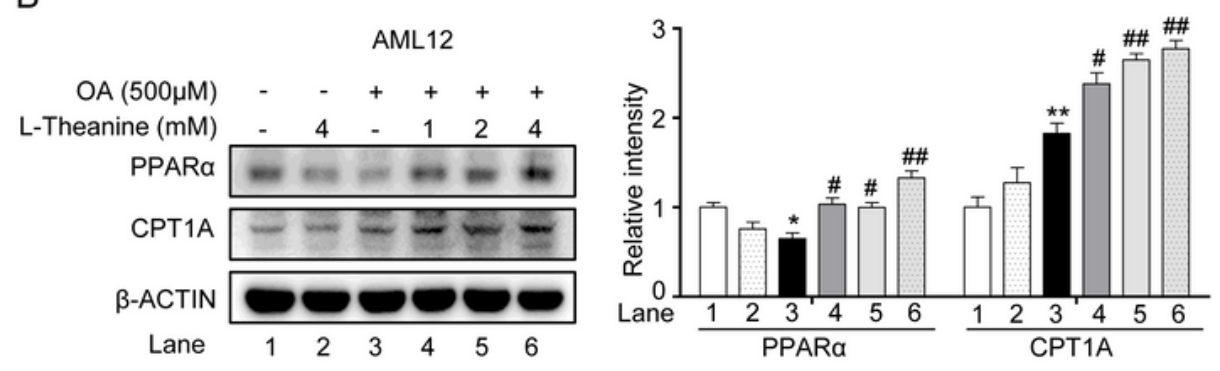

C
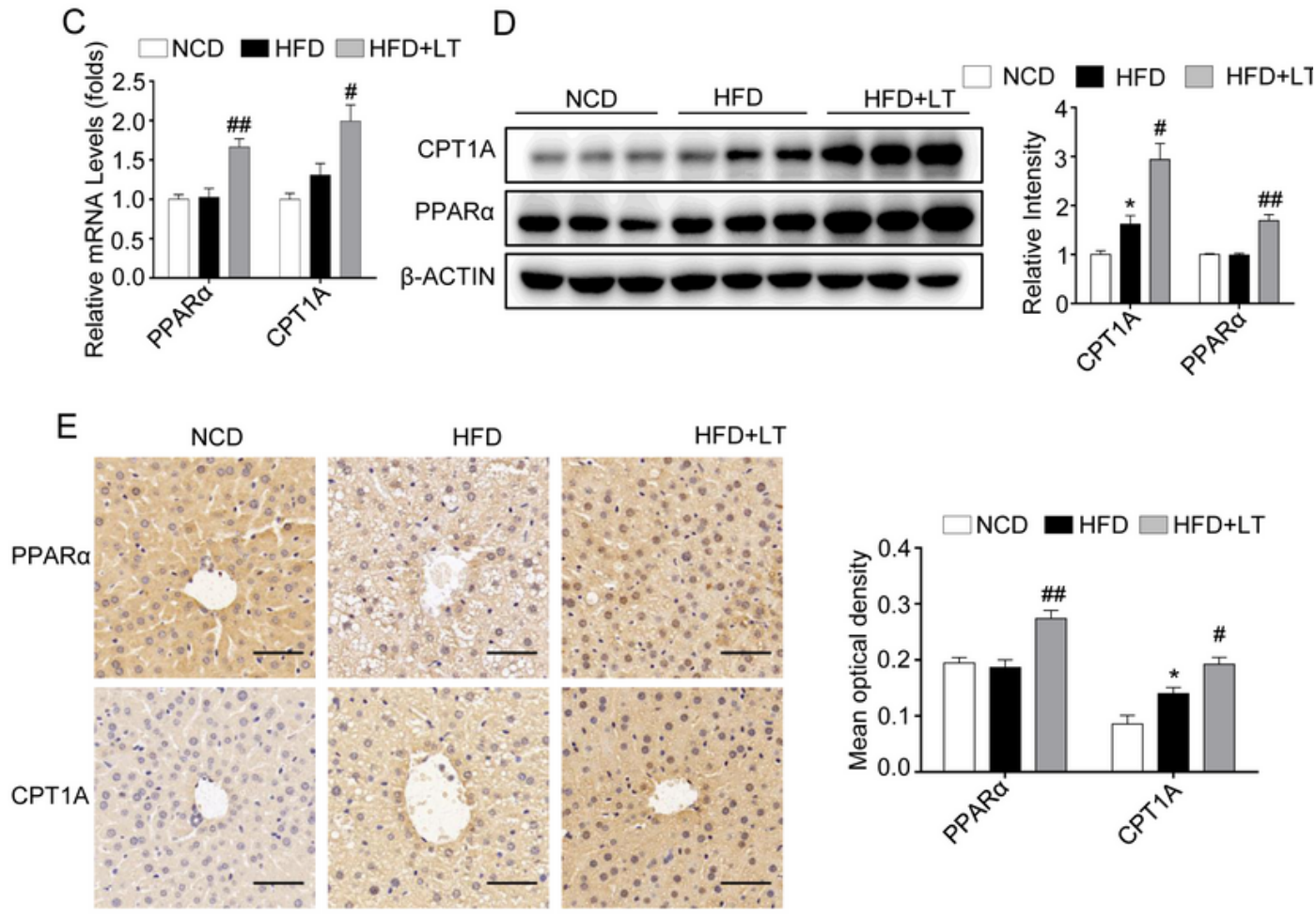

Figure 6

L-theanine promotes fatty acid $\beta$ oxidation through promoting the expressions of PPARa and CPT1A. HepG2 and AML12 cells were treated with 500 uM OA for $24 \mathrm{~h}$ with or without pretreated L-theanine for 
2h. Western blot analysis showing protein expression of PPARa and CPT1A in HepG2 (A) and AML12 (B) cells. C57BL/6J mice were fed normal control diet (NCD), HFD or HFD supplemented with L-Theanine for 16 weeks $(n=8)$. (C) mRNA expression of PPARa and CPT1A in mice liver. (D) Protein levels of PPARa and CPT1A in mice liver. (E) Representative images of immunohistochemical staining of PPARa and CPT1A in liver sections. Band intensity was quantified by densitometry analysis. Values are expressed as mean \pm SEM deviation of three independent experiments. ${ }^{*} p<0.05,{ }^{*} p<0.01$ VS control or NCD group; $\# p<0.05, \# \# p<0.01$ VS OA or HFD group. Scale bar $=50 \mu \mathrm{m}$. Abbreviation: LT, L-Theanine.

A
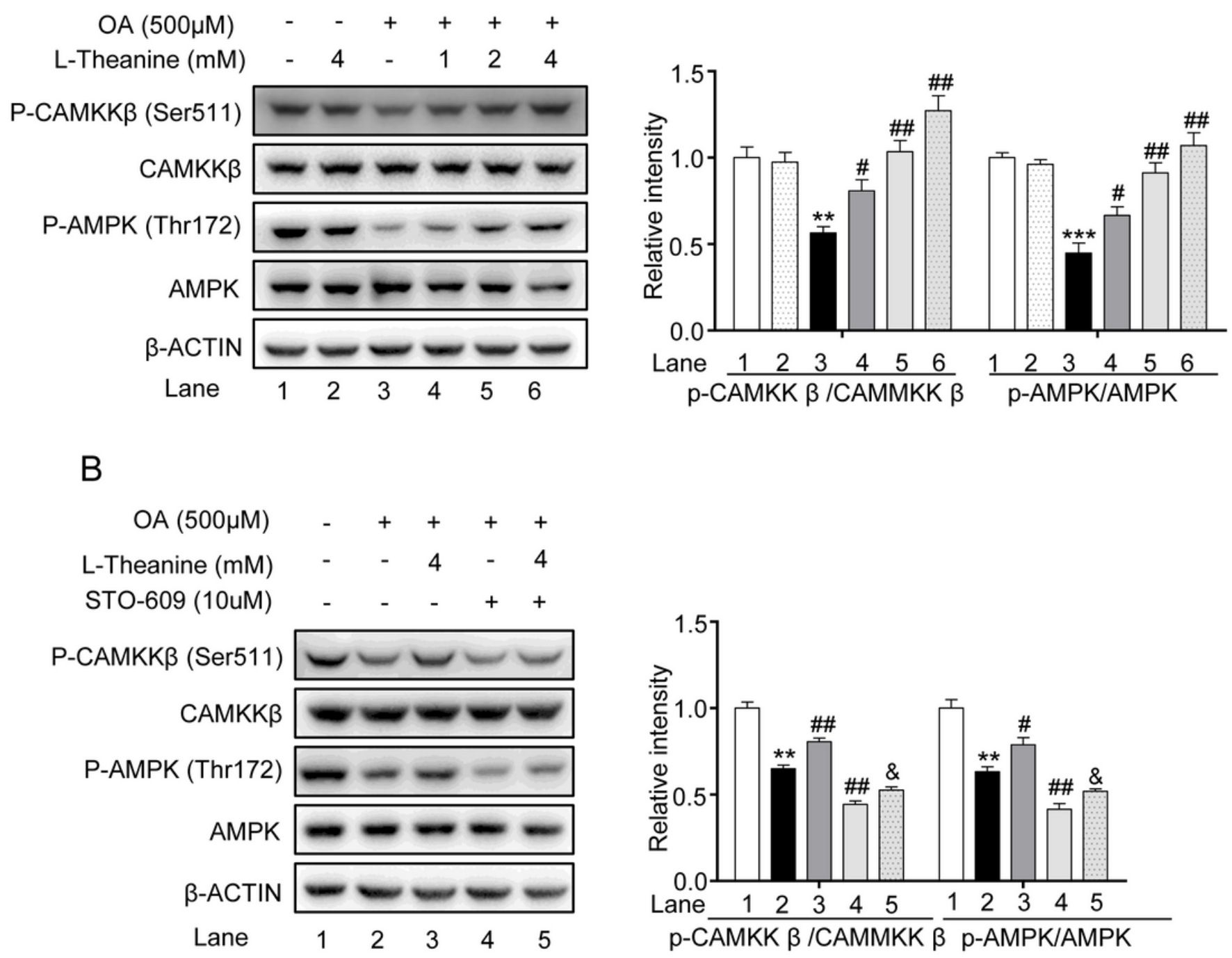

Figure 7

L-theanine regulates hepatocyte lipid metabolic pathways via the CaMKK $\beta$-AMPK signaling pathway. HepG2 cells were treated with 500 uM OA for $24 \mathrm{~h}$ with or without pretreated L-theanine for $2 \mathrm{~h}$. (A) 
Western blot analysis showing protein expression of p-CaMKK $\beta$ and p-AMPK in HepG2 cells. HepG2 cells were pretreated with 10uM STO-609 for $2 \mathrm{~h}$, then added L-theanine co-incubated for $2 \mathrm{~h}$, finally, OA were added co-incubated for $24 \mathrm{~h}$. (B) Abrogating effect of CaMKK $\beta$ inhibitor on L-theanine-facilitated phosphorylation of CaMkk $\beta$ and AMPK $\alpha$ in HepG2 hepatocytes. Band intensity was quantified by densitometry analysis. Values are expressed as mean \pm SEM deviation of three independent experiments. ${ }^{\star} p<0.05,{ }^{\star \star} p<0.01,{ }^{\star \star \star} p<0.001$, VS control group; $\# p<0.05, \# \# p<0.01$, VS OA group; ${ }^{\star} p<0.05$ VS OA+STO-609 group. Abbreviation: LT, L-Theanine.

| $:$ supressed by L-theanine

$\longrightarrow$ : promoted by L-theanine

$\longrightarrow$ : Downregulated by L-theanine
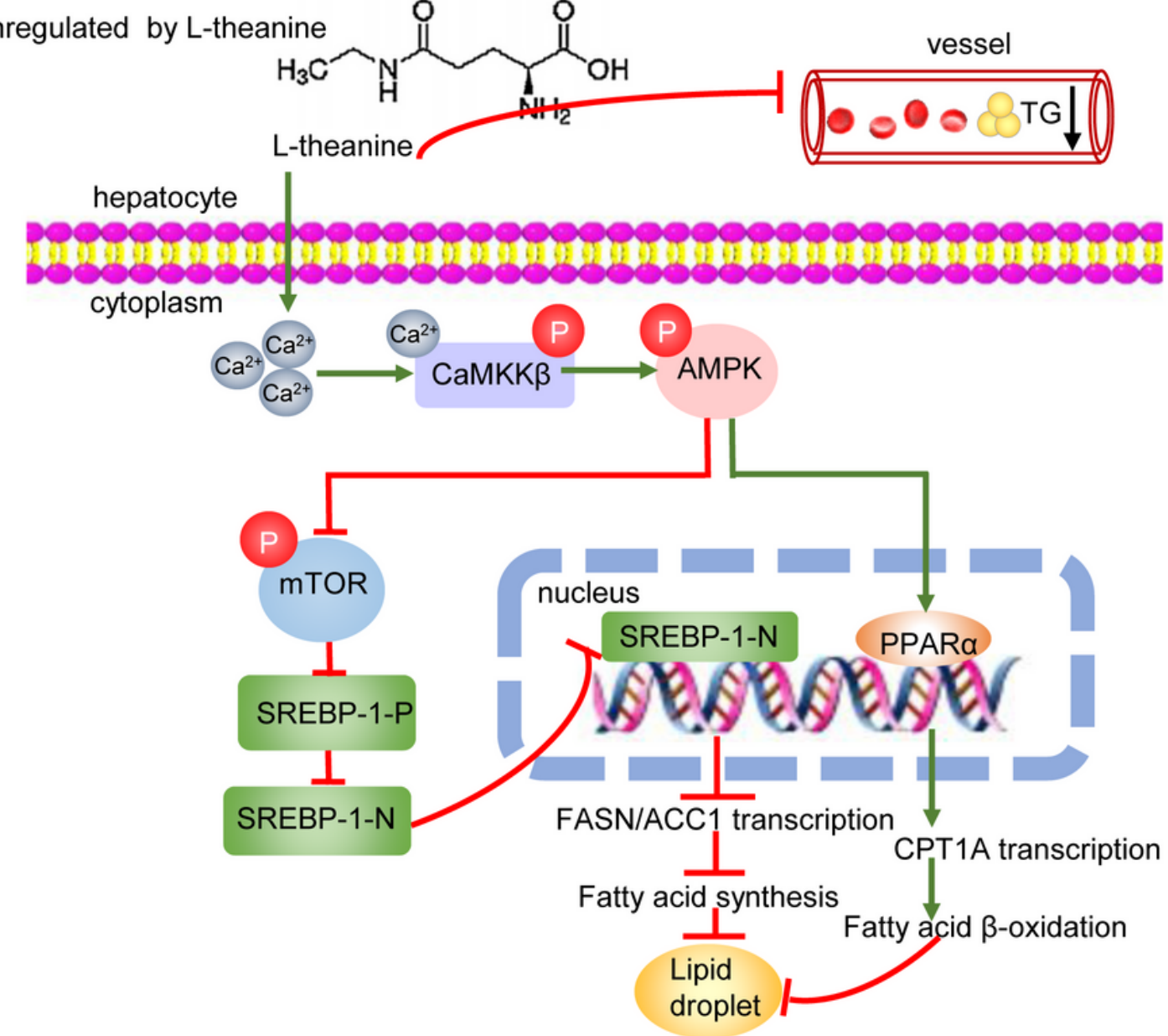

Figure 8

Schematic of the role of L-theanine in protecting against nonalcoholic hepatic steatosis.

\section{Supplementary Files}

This is a list of supplementary files associated with this preprint. Click to download. 
- SupplementaryFigure.S1.tif

- SupplementaryFigure.S2.tif

- SupplementaryFigure.S3.tif 